\title{
BMJ Open Cross-sectional prospective feasibility study of newborn screening for sickle cell anaemia and congenital hypothyroidism in Guyana
}

\author{
Bibi Areefa Alladin (D , , ${ }^{1}$ Pheona Mohamed-Rambaran, ${ }^{1}$ Vijay Grey, ${ }^{2}$ \\ Andrea Hunter, ${ }^{3}$ Pranesh Chakraborty, ${ }^{4}$ Matthew Henderson, ${ }^{4}$ Jennifer Milburn, ${ }^{4}$ \\ Laurie Tessier ${ }^{4}$
}

To cite: Alladin BA, MohamedRambaran P, Grey V, et al. Cross-sectional prospective feasibility study of newborn screening for sickle cell anaemia and congenital hypothyroidism in Guyana. BMJ Open 2022;12:e046240. doi:10.1136/ bmjopen-2020-046240

- Prepublication history for this paper is available online. To view these files, please visit the journal online (http://dx.doi org/10.1136/bmjopen-2020046240).

Received 24 October 2020 Accepted 04 February 2022
Check for updates

(c) Author(s) (or their employer(s)) 2022. Re-use permitted under CC BY-NC. No commercial re-use. See rights and permissions. Published by BMJ.

${ }^{1}$ Pediatrics, Georgetown Hospital, Georgetown, Guyana ${ }^{2}$ Laboratory Medicine, McMaster University, Hamilton, Ontario, Canada

${ }^{3}$ Pediatrics, McMaster University, Hamilton, Ontario, Canada ${ }^{4}$ Children's Hospital of Eastern Ontario, Ottawa, Ontario, Canada

Correspondence to

Dr Bibi Areefa Alladin;

bibialladin@yahoo.com

\section{ABSTRACT}

Introduction Newborn screening (NBS) is a test done shortly after birth to detect conditions that cause severe health problems if not treated early. An estimated $71 \%$ of babies worldwide are born in jurisdictions that do not have an established NBS programme. Guyana currently has no NBS programme and has established a partnership with Newborn Screening Ontario (NSO) to initiate screening. Objectives To assess the feasibility of implementing a NBS programme in Guyana for congenital hypothyroidism (CH) and haemoglobinopathies (HBG) and to report on screen positive rates and prevalence (Hardy-Weinberg equilibrium (HWE)) for $\mathrm{CH}$ and $\mathrm{HBG}$.

Methods Term, healthy Guyanese infants were evaluated (with consent) using heel prick dried blood spots (DBS) shortly after birth (closer to 24 hours of life). DBS samples were analysed at NSO. Screening test for $\mathrm{CH}$ was done using a human thyroid-stimulating hormone (hTSH) assay. Mean hTSH levels between the Guyanese sample and the Ontarian population were compared using Student's t-test with an alpha of 0.05 . Screening test for HBG was performed with a cation-exchange high-performance liquid chromatography.

Results The pilot was conducted from 6 June 2016 to 22 September 2017. Georgetown Public Hospital Corporation recruited 2294 mothers/infants. Screen positive rate for $\mathrm{CH}$ in our sample was $0.0 \%(0 / 2038$ infants). Mean TSH levels in Guyanese samples ( $1.7 \mu \mathrm{U} / \mathrm{mL}$ blood) was noticed to be significantly different than in the Ontarian population (4.3 $\mu \mathrm{U} / \mathrm{mL}$ blood) $(p<0.05)$. Screen positive rate for sickle cell anaemia (SCA) in our sample was $0.3 \%$ (7/2039 patients), and the carrier rate was $8.4 \%$ (172/2039 patients). Using the HWE, the SCA frequency (S allele frequency) ${ }^{2}$ is $0.049^{2}=0.002$

Conclusion NBS for CH and SCA in Guyana could be beneficial. Future work should focus on conducting larger pilots which could be used to inform diagnosis and treatment guidelines for Guyanese people.

\section{INTRODUCTION}

Newborn screening (NBS) is a screening test done shortly after birth to detect conditions that cause severe health problems if not treated early. ${ }^{1}$ NBS programmes have
Strengths and limitations of this study

- This first-time study on newborn screening in Guyana provides baseline data on sickle cell anaemia and thyroid hormone levels.

- The findings can be used by policymakers to justify future work, including policies for newborn screening in Guyana.

- The absence of previous studies prevented comparison and interpretation of thyroid hormone levels in the neonates.

- The small sample size and single-centre study prevents generalisation of findings to the Guyanese population.

- Difficulties were encountered with following up of participants with borderline or positive results due to their geographic locations.

been existing for over 50 years in developed countries. ${ }^{2}$ It allows clinical providers to identify infants at high risk for those conditions, provide diagnostic testing and initiate treatment to avoid severe outcome and to prevent death. ${ }^{3}$ Conditions included in NBS need to have a relatively high prevalence and their testing and treatment need to be simple, inexpensive and effective. ${ }^{2}$ While the number of conditions included in NBS panels varies across jurisdictions, the most advanced laboratory settings give NBS programmes the capability for screening for over 50 conditions. ${ }^{3}$

Centralisation of testing and advanced analytical methods such as tandem mass spectrometry which has increased the sensitivity and rapidity in evaluation have contributed to the progress made with screening neonates . ${ }^{4}$ In lower-income and middle-income jurisdictions, barriers such as lack of resources and infrastructures have limited the development of NBS programmes. ${ }^{4}$ Approximately $71 \%$ of babies worldwide are born in a jurisdiction 
that does not have an established NBS programme. ${ }^{2}$ As such, the majority of infants born worldwide with diseases for which NBS exists do not get diagnosed and treated early. This leads to significant mortality, morbidity and economic burden of NBS-eligible diseases in jurisdictions where NBS is not developed. ${ }^{2}$

Guyana currently has no NBS programme and has established a partnership with Newborn Screening Ontario (NSO) to initiate screening. NSO was established in 2006 and coordinates NBS for the province of Ontario, Canada. NSO screens over 140000 newborns per year for 29 targeted diseases by collecting and analysing dried blood spot (DBS) samples in the first days of life. Congenital hypothyroidism $(\mathrm{CH})$ and sickle cell anaemia (SCA) are two relatively common conditions, which are good candidates for NBS and offered by NSO. In the Guyanese population, the prevalence of $\mathrm{CH}$ and SCA are unknown but there is evidence that they both have a significant public health burden based on the number of paediatric patients requiring care with SCA and hypothyroidism as outpatients and inpatients, making these two conditions eligible for a NBS pilot in Guyana.

$\mathrm{CH}$ is an endocrine condition that can result in growth deficiency and severe intellectual disability if left untreated. ${ }^{1}$ It is one of the most common preventable cause of intellectual disability with an incidence in newborns between 1:2000 and 1:4000, and as high as 1:600 newborns in iodine-deficient areas. ${ }^{1} \mathrm{CH}$ is most often permanent and is caused by an abnormality in thyroid gland development or a defect in thyroid hormonogenesis. ${ }^{5}$ In some cases, the condition is transient, in which can be due to the transplacental passage of maternal medications, maternal blocking antibodies, iodine deficiency or excess. ${ }^{5}$ Treatment for $\mathrm{CH}$ is effective, inexpensive and easy to manage for families. ${ }^{1}$ Infants diagnosed with $\mathrm{CH}$ near birth can start treatment and avoid any growth delay or intellectual disability and develop normally. ${ }^{1}$ However, when patients with $\mathrm{CH}$ are identified later, treatment does not suffice to eliminate developmental and growth delays resulting from $\mathrm{CH}$, which underlines the importance of NBS for $\mathrm{CH}^{1}{ }^{1} \mathrm{CH}$ was added to NBS programmes starting in the mid-1970s, and has shown to be effective in reducing morbidity, mortality and the economic burden of $\mathrm{CH}$ : the jurisdictions screening for $\mathrm{CH}$ have eliminated neurodevelopmental impairments resulting from $\mathrm{CH}^{2}{ }^{2}$ Since establishing NBS for $\mathrm{CH}$, Cuba, Puerto Rico and the US Virgin Island have been screening more than $95 \%$ of their population for $\mathrm{CH}$ and reported a birth incidence ranging from 1: 3600 and 1: 9526. ${ }^{2}$ In Brazil, the $\mathrm{CH}$ prevalence has been reported as $1: 2259 .^{2}$ The $\mathrm{CH}$ prevalence in Guyana remains unknown.

In Guyana, SCA is among the leading causes for admission to paediatric wards. During a 1-year study, it accounted for 102 of 1380 admissions. ${ }^{6}$ SCA is a group of autosomal recessive inherited conditions that affects the formation of haemoglobin and the functioning of red blood cells. The gene mutations responsible for SCA are most common in populations of African, South
Asian, Middle Eastern and Mediterranean origins. ${ }^{7}$ SCA is one of the most common genetic conditions worldwide. The global birth prevalence of homozygous SCA is estimated to be 111.91 per 100000 live births with disparities by region. Africa has a birth prevalence of 1125.49 per 100000 compared with 43.12 per 100000 in Europe. ${ }^{8}$

SCA is a global health public concern, a survey conducted by the WHO reported that haemoglobin disorders such as SCA are a public health concern for $71 \%$ of the 229 countries surveyed, which represent $89 \%$ of births worldwide. ${ }^{9}$

While the prevalence of SCA in Guyana in unknown, data are available for some Caribbean countries through NBS. Jamaica has the highest prevalence of SCA at $0.65 \%$, followed by Grenada $(0.63 \%)$, Haiti $(0.58 \%)$ and Tobago $(0.57 \%)$. Trait prevalence was highest in Jamaica at $14 \%$ and St. Vincent and Grenadines (15\%), followed by St. Lucia $(13.8 \%)$, Haiti $(13.5 \%)$ and Tobago $(13.2 \%) .{ }^{10}$

Patients with sickle cell disease (SCD) experience a wide range of adverse outcomes, including, but not limited to anaemia, stroke, ischaemic organ damage, pain crises, chronic respiratory disease, recurrent infections and death. ${ }^{11}$ In 2008, the United Nations (UN) recognised SCA, a form of SCD, as a public health concern and urged relevant parties to strengthen health systems and primary care delivery for SCA. ${ }^{12}$ Since this declaration, many jurisdictions have recognised the need for SCA NBS in order to enable early identification and treatment of SCA affected infants. ${ }^{12}$ Several studies have concluded that NBS for SCA and early treatment for SCA leads to reduced morbidity and mortality. Notably, a study conducted in the Republic of Benin found that establishment of a NBS for SCA has led to a mortality rate 10 times lower for children under 5 years of age. ${ }^{13}$ In North America, SCA mortality for children under 5 years of age has been almost eliminated thanks to early identification and treatment from NBS. ${ }^{14}$

The Georgetown Public Hospital Corporation (GPHC) partnered with NSO to conduct a pilot study of NBS for $\mathrm{CH}$ and SCA in Guyana. Given the infrastructure challenges for establishing and delivering NBS in Guyana, partnering with an established NBS programme could help overcome obstacles and help provide data that support the implementation of a NBS programme, such as disease prevalence and the NBS test's positive predictive values in the Guyanese population.

\section{Objectives}

The objectives of this pilot NBS study were:

- To assess the feasibility of implementing a NBS programme in Guyana for $\mathrm{CH}$ and haemoglobinopathies (HBG).

- To assess the potential benefits of implementing a NBS programme in Guyana for $\mathrm{CH}$ and HBG.

- To report on screen positive rates for $\mathrm{CH}$ and HBG (SCD and traits) for the Guyanese population. 
- To report on HBG prevalence in Guyana based on NBS results and using the Hardy-Weinberg equilibrium $(\mathrm{HWE})$.

\section{METHODS}

\section{Study design}

This pilot project was a prospective descriptive study done at GPHC from 6 June 2016 to 22 September 2017.

\section{Inclusion criteria}

Term, healthy babies (gestational age of 37 weeks or later) born at GPHC during the study period whose mothers provided written consent after being briefed on the study.

\section{Exclusion criteria}

Babies with sepsis, and those admitted to the neonatal intensive care unit were excluded since their mothers were potentially under undue stress and may not have consented. Additionally, the sampling of these babies may have created additional exposure to personnel who can introduce infection and further compromise their immune system. Further, sick newborns can have abnormal TSH due to their illness which may not be indicative of $\mathrm{CH}$ and could have cause a bias in the findings.

\section{Sample size}

The sample size was calculated to be 3250 neonates based on the number of babies born annually, which represented the duration of funding. Guyana has an average of 15000 live births per year with 22\% being born at GPHC. It was not feasible to include rural hospitals in this pilot study.

\section{Sample collection and handling}

Newborn babies were tested after mothers were given a written document and a brief discussion of the purpose of the research, the risks and benefits of being tested and the potential complications from a heel prick DBS sample collection. A written consent form was provided to mothers for signature. Some mothers requested that the fathers' consent as well. Only babies whose parents consented were included in the study. Data were coded with a unique identifier.

A DBS sample was collected by trained laboratory technicians via heel prick and using a filter card shortly after birth (closer to 24 hours of life). These samples were stored at the Medical Laboratory at GPHC according to standard protocols for storing DBS. DBS filter cards were air shipped (at room temperature) weekly to NSO for analysis, with a shipping turnaround time of 3 days.

\section{Analysis}

Samples were analysed for $\mathrm{CH}$ and SCA on the day of receipt by NSO, and the NBS results were sent from NSO to GPHC within 14 days of samples receipt. NBS results were categorised as screen positive (higher probability that the infant is affected by the disease and confirmatory diagnosis testing is needed), screen negative (low probability that the infant is affected by the disease and no follow-up is needed), or unsatisfactory (the sample quality did not permit the NBS test). Confirmatory testing and follow-up care were planned at GPHC.

\section{Congenital hypothyroidism}

The screening test for $\mathrm{CH}$ was done using a human thyroid-stimulating hormone (hTSH) assay. The neonatal hTSH assay is a solid phase, two-site fluoroimmunometric assay based on the direct sandwich technique. Specimens containing hTSH are reacted with anti-hTSH IgG, coated on the wells of the microtitre plates and europiumlabelled anti- hTSH IgG (Eu tracer). The wash buffer elutes hTSH from the DBS. The enhancement solution dissociates the europium ions from the labelled antibody where they form highly fluorescent chelates. The fluorescence measured in each well is proportional to the concentration of hTSH in the sample. If the initial hTSH level in a sample was found to be greater than or equal to $13 \mu \mathrm{U} / \mathrm{mL}$ of blood, a confirmatory hTSH measurement was made using the same technique outlined above. If the confirmatory hTSH level was found to be greater than or equal to $17 \mu \mathrm{U} / \mathrm{mL}$ of blood, the newborn was considered screen positive for $\mathrm{CH}$. Otherwise, the patient is categorised to be screen negative for CH. Mean hTSH levels between the Guyanese sample and the Ontarian population were compared using Student's t-test with an alpha of 0.05 .

\section{Sickle cell anaemia}

The screening test for SCA was performed with a cationexchange high-performance liquid chromatography used to separate the different variants of haemoglobin present in a DBS elutes (reconstituted DBS). Using a two dualpiston pump, the ionic strength of the buffer is increased, encouraging the more strongly retained haemoglobin to elute from the column. Each haemoglobin has a characteristic retention time. The instrument quantifies the amount of haemoglobin variants present in the sample. These include adult haemoglobins (A, A2) which are the most common types in the normal population; fetal haemoglobins (F, F1) and minor haemoglobins (E, S, C). Together, the quantitative haemoglobin results (in the form of peaks in the chromatogram) are interpreted by a clinician who assigns the chromatogram a single haemoglobin pattern determination. The haemoglobin pattern determination was used to determine if the newborn was screen negative or screen positive for SCA. This NBS tests allows for identification of carriers of SCA.

$\mathrm{S}$ allele frequency was calculated using the below equation (equation 1):

$$
\begin{aligned}
& \text { S allele frequency }=(\text { number of carriers })+ \\
& (2 \times(\text { number of screen positive }) \\
& /(\text { number of infants screened } \times 2)
\end{aligned}
$$

SCA prevalence in Guyana was estimated using a population of approximately 746955 (according to a census conducted in 2012 conducted by the Bureau of Statistics of Guyana). ${ }^{13}$ The Bureau of Statistics Guyana estimated 
Table 1 Wilson-Jungner principles ${ }^{18}$

\section{Principle}

\section{The condition sought should be an important health problem}

2. There should be an accepted treatment for patients with recognised diseases

3. Facilities for diagnosis and treatment should be available

4. There should be a recognisable latent or early symptomatic phase

\begin{tabular}{|c|c|}
\hline 5. There should be a suitable test or examination & Yes \\
\hline 6. The test should be acceptable to the population & Yes \\
\hline $\begin{array}{l}\text { 7. The natural history of the condition, includ } \\
\text { from latent to declared disease, should be ur }\end{array}$ & Yes \\
\hline
\end{tabular}
from latent to declared disease, should be understood

8. There should be an agreed policy on whom to treat as patients Health, Guyana 9. The cost of case-finding (including diagnosis and treatment of Yes patients) diagnosed should be economically balanced in relation to possible expenditure on medical care as a whole

10. Case-finding should be a continuing process and not a 'once and for all' project

\section{Principle met?}

Yes

Yes

Available but was not assessed in this pilot. GPHC can diagnose and treat SCA and $\mathrm{CH}$.

Yes

Yes

Yes

Draft document awaiting final approval by Ministry of

Yes

-policy brief for implementation of NBS was presented to the Pan American Heath Organization (PAHO) Guyana office in $\mathbf{2 0 2 0}$ for implementation of routine NBS. No further work on this due to COVID-19 pandemic.

$\mathrm{CH}$, congenital hypothyroidism; GPHC, Georgetown Public Hospital Corporation; SCA, sickle cell anaemia.

the annual projected births to be between 13963 and 15864 in the years between 2000 and 2025 in Guyana, which was used to calculate the birth prevalence of SCA. ${ }^{15}$

\section{Outcome measures}

Primary outcomes were to report on screen positive rates for $\mathrm{CH}$ and $\mathrm{HBG}$ for the Guyanese population using HWE and percent positivity rates. The HWE is commonly used to estimate the frequency of a phenotype in a population, for example a recessive condition like SCA, because sample sizes in cross-sectional studies are usually too small to allow for a precise estimate ${ }^{916}$ For $\mathrm{CH}$, mean hTSH levels and mean difference between the Guyanese sample and the Ontarian population were compared using Student's t-test with an alpha of 0.05.

Secondary outcome was to assess the feasibility of $\mathrm{CH}$ and SCA NBS as a public health programme in Guyana using Wilson-Jungner criteria as the outcome measure (as shown in table 1 ).

\section{Patient and public involvement}

No patients or member of the public were included in the design of this study, patients were included in the data collection phase and in the reporting of results that needed follow-up. Patients were recruited as the sample population after sensitisation of their mothers.

\section{RESULTS}

\section{Pilot experience and feasibility}

The pilot was conducted from 6 June 2016 to 22 September 2017. GPHC recruited 2294 mothers/infants.
There were no reported harms to babies who were assessed. All samples were successfully shipped to NSO. Confirmation of diagnosis of the screen positive neonates was not obtained since telephone numbers provided were unreachable and mothers failed to bring in their child, so this report is solely on the screen positive rate for $\mathrm{CH}$ and SCA, and on the estimated prevalence of SCA assuming HWE. Of note, one mother followed up and her child was confirmed as SCA.

\section{Congenital hypothyroidism}

Of the 2294 received samples, 256 were excluded from CH NBS testing as they were determined unsatisfactory for testing (figure 1). The most common reason for exclusion was an insufficient blood quantity of the sample. The screen positive rate for $\mathrm{CH}$ in our sample was $0.00 \%$ (0/2038 infants). Mean TSH levels in Guyanese samples

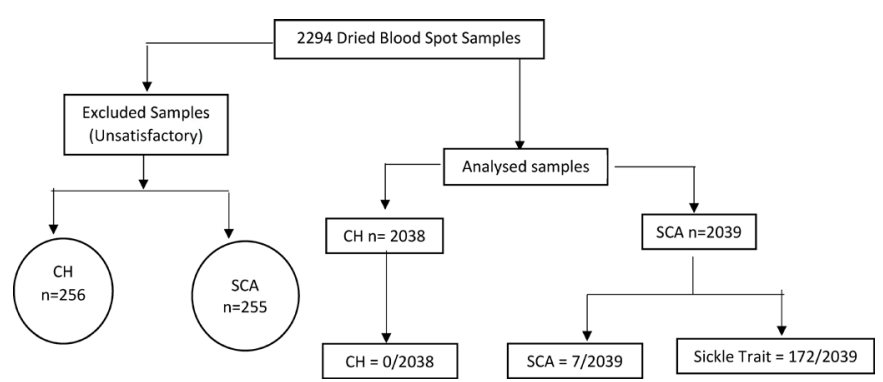

Figure 1 Flow diagram of sample collection and results. Newborn screening dried blood spot sample collection, and primary results for $\mathrm{CH}, \mathrm{SCA}$ and sickle cell trait. $\mathrm{CH}$, congenital hypothyroidism; SCA, sickle cell anaemia. 
Table 2 SCA genotype and S allele frequencies in the study sample

\begin{tabular}{llll} 
AA frequency-screen negative (\%) & AS frequency-carriers (\%) & SS frequency-screen positive & S allele frequency \\
\hline 51.35 & 9.07 & 0.34 & 0.049
\end{tabular}

Using the HWE, the SCA frequency is $(S \text { allele frequency })^{2}=0.049^{2}=0.002$.

HWE, Hardy-Weinberg equilibrium; SCA, sickle cell anaemia.

$(1.70 \mu \mathrm{IU} / \mathrm{mL}$ blood) was significantly different $(\mathrm{p}<0.05)$ than in the Ontarian population $(4.30 \mu \mathrm{IU} / \mathrm{mL}$ blood $)$.

\section{Sickle cell anaemia}

Of the 2294 received samples, 255 were excluded of SCA NBS testing as they were determined unsatisfactory for testing (figure 1). The most common reason for exclusion was insufficient blood quantity of the sample. The screen positive rate for SCA in our sample was $0.30 \%(7 / 2039$ patients), and the carrier rate was $8.41 \%(172 / 2039$ patients). As we were unable to obtain follow-up diagnostic data for all the screen positive infants, the true positive rate and the positive predictive value of this SCA NBS test in the Guyanese population could not be ascertained. However, as this NBS test identifies SCA-affected infants as well as carriers, the allele frequency for SCA in the study sample and estimate of SCA prevalence in the Guyanese population using the HWE, assuming sample is representative of the population in terms of allele frequency, could be deduced. Table 2 presents the genotype and $\mathrm{S}$ allele frequencies in the study sample; the $\mathrm{S}$ allele frequency being represented by equation 1 in Methods section.

Using the projected estimate birth value for 2020 of 15126 births, it can be estimated that the birth prevalence of SCA in Guyana is 30 births per year $(0.2 \%)$.

\section{CH and SCA as NBS candidate diseases in Guyana as per Wilson-Jungner criteria}

To further assess the suitability of $\mathrm{CH}$ and SCA NBS as a public health programme in Guyana, mapping of Guyana's experience was done onto the Wilson-Jungner criteria from table 1 . These criteria were established in 1968 in a report for the $\mathrm{WHO}^{17}$ and have been developed as an evaluation framework to determine the eligibility of a given condition and its test for a screening programme. The Wilson-Jungner principle have been widely used as a checklist to establish NBS programmes. ${ }^{18}$ The principles acknowledge the technical characteristics of a given NBS test as well the significance of political, economic, social and health issues of an NBS test or programme. ${ }^{18}$ Table 1 presents the 10 Wilson-Jungner principles and whether each principle was met in this pilot study. Seven out of the 10 principles were met in the study. Principle 3 was not assessed in this pilot but is met locally. Principles 8 and 10 were being addressed prior to the pandemic and is currently on hold due to prioritisation of resources.

\section{DISCUSSION}

A pilot study of NBS in Guyana was conducted in which a remote laboratory performed the screening tests. The pilot has shed light on the feasibility and potential benefits of NBS for CH and SCA in the Guyanese population. This pilot study also provided an estimate of the SCA prevalence and birth prevalence in Guyana.

There were no screen positive infants for $\mathrm{CH}$ in the sample population, but the researchers are familiar with cases of CH in Guyana. Small sample population of 2294 was responsible for zero positivity for $\mathrm{CH}$ as incidence ranges from 1:2000 to 1:4000. At least 4000-5000 babies will need screening for one positive sample. ${ }^{1}$ Currently, children are evaluated for hypothyroidism in Guyana if there is a high index of suspicion such as developmental delay. Late diagnosis and intervention have adverse prognostic factors for these children. Further research is needed to determine the prevalence of $\mathrm{CH}$ in newborns and the potential causes of the mean TSH levels difference between Guyana and Ontario, and whether TSH cutoffs for CH NBS in Guyana must be adjusted accordingly. It would notably be important to determine whether the shipment time and conditions (temperature, humidity) from Guyana to Ontario, Canada affect TSH levels in the DBS, or if mean TSH levels are different in the Guyanese population due to other factors specific to this population. It is possible that some infants in the study sample are in fact affected by $\mathrm{CH}$ with TSH levels higher than the mean TSH level for the sample population. Diagnosis of $\mathrm{CH}$ could not have been confirmed due to lack of follow-up data. Confirming diagnosis would further help determine if the TSH cut-off for CH NBS tests in Guyana should be different than the one used in Ontario by NSO. Currently, we are unable to comment on recommending $\mathrm{CH}$ as a priority NBS in Guyana.

The sample's screen positive rate for SCA $(0.3 \%)$, as well as the estimated SCA allele frequency and birth prevalence of SCA in Guyana assuming HWE $(0.2 \%)$ are lower than what has been reported in some Caribbean jurisdictions. ${ }^{10}$ This may be due to small sample size, insufficient statistical power and insufficient external validity of the study sample, and centralised testing at GPHC without having a good representation of the rural areas in the sample population as SCA is higher in Afro-Caribbean people. To our knowledge, this is the first report of an estimated prevalence and birth prevalence of SCA in Guyana, and further research is needed to clarify these findings. The estimated birth prevalence from this study suggests that about 30 newborns per year in Guyana are 
affected by SCA, which indicates that Guyana may benefit from SCA NBS. Guyana recently concluded a draft document for the management of SCA and Thalassaemia and there is an increase in specialty service for this population. Guyana has a significant East Indian ancestry with genetic susceptibility to Thalassaemia, this population may benefit from HGB screening that may identify beta Thalassaemia.

The eligibility of CH and SCA NBS in Guyana is further supported by our assessment of this pilot's experience using the Wilson-Jungner principles. Only 3 of the 10 principles have not been met in this study. These three unmet principles were out of the study's scope and could be established in future work. Principle 3, Facilities for diagnosis and treatment should be available, Principle 8, There should be an agreed policy on whom to treat as patients and Principle 10, Case-finding should be a continuing process and not a 'once and for all' project, could be met through some policy work in Guyana. Bibi A Alladin has developed a policy brief for the implementation of NBS for HGB in Guyana under the guidance of Pan American Health Organization.

Overall, our experience suggests that NBS for $\mathrm{CH}$ and SCA in Guyana could be beneficial, and that it is feasible to establish a programme by shipping DBS samples to a remote NBS lab for analysis while Guyana builds capacity for local testing. Over the last 3 years, Guyana has dispatched more doctors to the rural areas as the medical capacity continues to grow, this may help to mitigate the challenges with following up of screen positive samples from rural areas through the network of primary healthcare.

Future work should focus on conducting larger pilots that would allow more diagnostic data to be collected, which in turn would allow a more accurate estimate of positive predictive values of the NBS tests and a better estimation of the mortality and morbidity reduction in Guyana if NBS is implemented. Additionally, these findings could be used to inform diagnosis and treatment guidelines for Guyanese people.

\section{Limitations}

Lack of access to testing facilities in Guyana was a major hinderance to the start of this project until NSO became involved. Only children born centrally were included in the study due to lack of resources and access to rural areas. Sample population may not be a true representation of the Guyanese population distribution. There were no positive cases of $\mathrm{CH}$ due to small sample size as $\mathrm{CH}$ prevalence ranges from 1:2000 to 1:9000. Larger studies with more robust methods for diagnostic follow-up are needed to establish prevalence of $\mathrm{CH}$ in newborns in Guyana, limited funding did not permit a larger study at this time.

Sample rejection was $11 \%$, this was due to lack of familiarity of DBS as a routine method of sample collection. Technicians were trained for DBS, but skill set varied among technicians. This further reduced our sample size for the analysis.

We were unable to follow-up most of the screen positive cases for SCA despite many attempts, as these cases were all from the rural areas and contact information was not reliable.

Acknowledgements We would like to thank Guyana Bank for Trading and Industry (GBTI) for funding this project as part of a public-private partnership, and Georgetown Public Hospital Corporation (GPHC) for providing much needed resources. Retired Major General Joseph Singh was instrumental in securing funding for this project. We would like to acknowledge the Laboratory Technicians and Medical Technologists at GPHC who volunteered their time for sample collection. Jennifer McKnight provided information on NBS in the Caribbean and has volunteered to assist with implementation of NBS in Guyana.

Contributors BAA, PM-R, VG and AH developed the proposal. BAA and PM-R provided resources and supervision for sample collection, shipment and follow up of results locally, including reporting and patient contact and completed the final manuscript and article submission. PC, MH, JM and LT contributed to the methodology, provided resources for analysing samples, interpretation of the results and assisted with compilation of the manuscript. BAA secured funding and is acting as guarantor.

Disclaimer This project was funded by Guyana Bank for Trading and Industry (GBTI) through a public-private partnership. Funds were used to purchase supplies for sample collection and for testing. Georgetown Public Hospital Corporation (GPHC) paid for the shipment of samples to NSO weekly and provided human resources for sample collection. The funder was not involved in the research or publication.

\section{Competing interests None declared.}

Patient and public involvement Patients and/or the public were involved in the design, or conduct, or reporting or dissemination plans of this research. Refer to the Methods section for further details.

\section{Patient consent for publication Consent obtained from parent(s)/guardian(s)}

Ethics approval This study involves human participants and was approved by Guyana Ministry of Public Health Institutional Review Board (IRB \#225) and by the Children's Hospital of Eastern Ontario Research Ethics Board (REB Protocol No: 17/210X). Participants gave informed consent to participate in the study before taking part.

Provenance and peer review Not commissioned; externally peer reviewed.

Data availability statement Data are available upon reasonable request. Data may be obtained from a third party and are not publicly available. Data are available from Pheona Mohamed-Rambarran at Georgetown Hospital by emailing pheonar@ yahoo.com.

Open access This is an open access article distributed in accordance with the Creative Commons Attribution Non Commercial (CC BY-NC 4.0) license, which permits others to distribute, remix, adapt, build upon this work non-commercially, and license their derivative works on different terms, provided the original work is properly cited, appropriate credit is given, any changes made indicated, and the use is non-commercial. See: http://creativecommons.org/licenses/by-nc/4.0/.

\section{ORCID iD}

Bibi Areefa Alladin http://orcid.org/0000-0002-6724-5606

\section{REFERENCES}

1 International Atomic Energy. Screening of newborns for congenital hypothyroidism. Vienna, 2005.

2 Ford G, LaFranchi SH. Screening for congenital hypothyroidism: a worldwide view of strategies. Best Pract Res Clin Endocrinol Metab 2014;28:175-87.

3 Therrell BL, Padilla CD, Loeber JG, et al. Current status of newborn screening worldwide: 2015. Semin Perinatol 2015;39:171-87.

4 Therrell BL, Padilla CD. Barriers to implementing sustainable national newborn screening in developing health systems. Int J Pediatr Adolesc Med 2014;1:49-60.

5 American Academy of Pediatrics, Rose SR, Section on Endocrinology and Committee on Genetics, American Thyroid 
Association, et al. Update of newborn screening and therapy for congenital hypothyroidism. Pediatrics 2006;117:2290-303.

6 Cameron L, Johnstone JC, Sparman A, et al. Guyana's paediatric training program: a global health partnership for medical education. Can Med Educ J 2017;8:e11-17.

7 van Heyningen AM, Levenston MJ, Tamminga N, et al. Estimated incidence of sickle-cell disease in Aruba and St. Maarten suggests cost-effectiveness of a universal screening programme for St. Maarten. West Indian Med J 2009;58:301-4.

$8 \mathrm{WHO} / \mathrm{TIF}$ Meeting on the Management of Haemoglobin Disorders (2007: Nicosia C, World Health Organization, Thalassaemia International Federation. Management of haemoglobin disorders: report of a joint WHO-TIF meeting, Nicosia, Cyprus, 16-18 November 2007. 2008;84

9 Modell B, Darlison M. Global epidemiology of haemoglobin disorders and derived service indicators. Bull World Health Organ 2008;86:480-7.

10 Knight-Madden J, Lee K, Elana G, et al. Newborn screening for sickle cell disease in the Caribbean: an update of the present situation and of the disease prevalence. Int J Neonatal Screen 2019;5:5.
11 Schnog JB, Duits AJ, Muskiet FAJ, et al. Sickle cell disease; a general overview. Neth J Med 2004;62:364-74.

12 Recognition of sickle-cell anaemia as a public health problem : resolution / adopted by the General Assembly, 2009. Available: http://dag.un.org/handle/11176/172628 [Accessed 20 Dec 2018].

13 Rahimy MC, Gangbo A, Ahouignan G, et al. Newborn screening for sickle cell disease in the Republic of Benin. J Clin Patho 2009. ;;62:46-8.

14 Grosse SD, Odame I, Atrash HK, et al. Sickle cell disease in Africa: a neglected cause of early childhood mortality. Am J Prev Med 2011;41:S398-405.

15 Bureau of Statistics - Guyana. Available: http://www.statisticsguyana. gov.gy/index.html [Accessed 19 Dec 2018].

16 ALLISON AC. The sickle-cell and haemoglobin C genes in some African populations. Ann Hum Genet 1956;21:67-89.

17 Raffle AE, Gray JAM, John AM. Screening : evidence and practice. Oxford University Press, 2007.

18 Jungner L, Jungner I, Engvall M, et al. Gunnar Jungner and the principles and practice of screening for disease. Int $J$ Neonatal Screen 2017;3:23 